\title{
Positron Lifetimes in Zirconia-Based Nanomaterials
}

\section{Procházka ${ }^{a}$, J. Č́́žEK ${ }^{a}$, J. Kuriplach $^{a}, \mathrm{O}$ Melikhova $^{a}$, T.E. Konstantinova ${ }^{b}$ And I.A. DANiLEnKo ${ }^{b}$}

${ }^{a}$ Charles University in Prague, Faculty of Mathematics and Physics

Department of Low Temperature Physics

V Holesovickach 2, 18000 Praha 8, Czech Republic

${ }^{b}$ Galkin Donetsk Institute for Physics and Engineering

National Academy of Science of Ukraine

Luxemburg Str., 83114 Donetsk, Ukraine

\begin{abstract}
Positron lifetime spectroscopy is employed in a comparative study of several zirconia-based materials: (i) the pressure-compacted nanopowders of the three zirconia polymorphs - pure $\mathrm{ZrO}_{2}$ (monoclinic), yttria-stabilized $\mathrm{ZrO}_{2}+3$ mol. $\% \mathrm{Y}_{2} \mathrm{O}_{3}$ (tetragonal) and yttria-stabilized $\mathrm{ZrO}_{2}+8$ mol. $\% \mathrm{Y}_{2} \mathrm{O}_{3}$ (cubic), (ii) ceramic materials obtained by sintering of the above two yttriastabilized zirconia nanopowders and (iii) the tetragonal and cubic yttria-stabilized zirconia monocrystals. Positron lifetime data observed on the nanopowders suggest that the two shortest components, exhibiting lifetimes of $\approx 180$ and $\approx 370 \mathrm{ps}$, arise from the annihilation of positrons trapped in defects associated with grain boundaries, presumably the vacancy-like defects and tripple points, respectively. Positron lifetime spectra observed on the ceramic materials resemble those found for the corresponding monocrystals, giving thus an additional support to the above interpretation of the nanopowders results.
\end{abstract}

PACS numbers: 78.70.Bj, 61.46.Hk, 81.05.Je, 81.07.Wx

\section{Introduction}

Zirconium dioxide (zirconia) is a base component of various materials suitable for a large area of industrial applications, for example, oxygen sensors, solid oxide fuel cells, refractory ceramics, machining tools, and grinding media. An exceptional combination of advantageous properties can be reached when zirconia-based materials are made of initial powders of nanometer grain size.

The pure zirconia is monoclinic at room temperature and has a high melting point of $\approx 2700^{\circ} \mathrm{C}[1]$. Its high-temperature applications are however limited due 
to a phase transformation to the denser tetragonal form at about $1100^{\circ} \mathrm{C}$ causing a large volume shrinkage and thus cracks within the material structure. A solid solution of zirconia with $\approx 8 \mathrm{~mol} . \%$ of $\mathrm{Y}_{2} \mathrm{O}_{3}$ (yttria) is of the cubic form with no transformation during heating up to $\approx 2500^{\circ} \mathrm{C}$. Such a solid solution is referred to as the fully yttria-stabilized zirconia (YSZ). For $\approx 3 \mathrm{~mol} . \%$ of yttria addition, the system is only partially stabilized, since the tetragonal phase formed after heating is metastable below $\approx 1000^{\circ} \mathrm{C}$.

The stabilization of zirconia by yttria leads to a violation of the stoichiometry and vacancies and vacancy-solute atom complexes are formed in the $\mathrm{ZrO}_{2}$ lattice. In the YSZ nanomaterials, grain boundaries (GB's) and defects associated to them (vacancy-like defects, triple points) become significant. In the materials originating from nanoparticles, voids and pores may occur as indicators of particle agglomeration and transformations during sintering. Thus a variety of open-volume structures of a size on a 1 to 100 nanometer scale can coexist with the zirconia-based nanomaterials. Such a situation is a challenge for positron annihilation spectroscopy (PAS). Despite of several earlier PAS investigations [2-5], there is still a lack of unambiguous explanation of PAS data on YSZ materials.

In the present work, the pressure-compacted and sintered YSZ nanopowders as well as respective monocrystalline YSZ's have been investigated by means of high-resolution positron lifetime (PL) spectroscopy, with main focus on the lifetimes below 0.5 ns. Results obtained on YSZ nanopowders have recently been reported in detail as a conference contribution [6]. The PL data observed in these materials of different complexity are compared and discussed from the point of defects involved.

\section{Experimental}

Specimens. The three zirconia-based nanopowders $[6,7]$ were used: (i) the pure $\mathrm{ZrO}_{2}$ (monoclinic phase, further referred to as $\mathrm{Z} 0 \mathrm{Y}$ ), (ii) the $\mathrm{ZrO}_{2}+3$ mol.\% $\mathrm{Y}_{2} \mathrm{O}_{3}$ (tetragonal, $\mathrm{Z} 3 \mathrm{Y}$ ) and (iii) the $\mathrm{ZrO}_{2}+8$ mol. $\% \mathrm{Y}_{2} \mathrm{O}_{3}$ (cubic, Z8Y). Powders were pressure-compacted under $250 \mathrm{MPa}$ at room temperature. The $\mathrm{Z} 3 \mathrm{Y}$ and Z8Y ceramic specimens were obtained by sintering the compacted nanopowders at $1200^{\circ} \mathrm{C} / 1 \mathrm{~h}$ and $1350^{\circ} \mathrm{C} / 1 \mathrm{~h}$, respectively. The YSZ monocrystals of tetragonal $\left(3 \mathrm{~mol} . \% \mathrm{Y}_{2} \mathrm{O}_{3}\right)$ and cubic $\left(9 \mathrm{~mol} . \% \mathrm{Y}_{2} \mathrm{O}_{3}\right)$ phase were studied by the PL technique, too.

$P L$ measurements. A $1.3 \mathrm{MBq}$ of a carrier-free ${ }^{22} \mathrm{Na}_{2} \mathrm{CO}_{3}$ water solution (iThemba Labs), dried and sealed between two $4 \mu \mathrm{m}$ mylarC foils (Dupont), was used as a positron source. The fast-fast configuration of a PL spectrometer described in Ref. [8] was employed in the present investigation. The spectrometer exhibited the time resolution of $163 \mathrm{ps}$ (FWHM) for the $1274-511 \mathrm{keV}$ coincidences. At least $10^{7}$ counts were accumulated in each PL spectrum. All the PL measurements were performed in air at room temperature. PL spectra were decomposed into the discrete components by means of the maximum likelihood 
method [8]. The positron annihilations in the source was measured using a well-annealed $\alpha$-iron and its intensity (total $I_{\text {source }} \approx 9 \%$ ) was recalculated to the particular materials under study following the method from Ref. [9].

\section{Results and discussion}

Up to four exponential components belonging to positron annihilations in the studied samples could be resolved in the measured PL spectra, besides of the para-positronium ( $p$-Ps) and positron source contributions. The $p$-Ps component $\left(\tau_{p-\mathrm{Ps}}\right.$ fixed at $\left.0.125 \mathrm{~ns}\right)$ was assumed to contribute by one third of the total ortho-positronium (o-Ps) intensity observed. Resulting lifetimes and relative intensities of the individual components $\left(\tau_{i}, I_{i}, i=1, \ldots, 4\right)$ were summarized in Table.

TABLE

Summary of positron lifetime results on zirconia-based materials studied in the present work (positron lifetimes $\tau_{i}$, intensities $I_{i}, \sum I_{i}=100 \%-I_{p-\mathrm{Ps}}$ ). The errors (one standard deviation) are given in parentheses in the units of the last significant digit. Sample abbreviations used in the 1st column are explained in the text.

\begin{tabular}{l|c|r|r|c|c|c|c|c|c}
\hline \hline \multicolumn{1}{c|}{ Sample } & $\tau_{1}[\mathrm{~ns}]$ & $I_{1}[\%]$ & $\tau_{2}[\mathrm{~ns}]$ & $I_{2}[\%]$ & $\tau_{3}[\mathrm{~ns}]$ & $I_{3}[\%]$ & $\tau_{4}[\mathrm{~ns}]$ & $I_{4}[\%]$ & $I_{1}: I_{2}$ \\
\hline powd. Z0Y & $0.189(2)$ & $45(1)$ & $0.373(4)$ & $44(1)$ & $2.0(1)$ & $1.54(5)$ & $34(2)$ & $7.4(3)$ & $1.03(5)$ \\
powd. Z3Y & $0.184(3)$ & $35(1)$ & $0.383(3)$ & $55(1)$ & $2.1(2)$ & $0.96(5)$ & $34(2)$ & $6.4(3)$ & $0.63(3)$ \\
powd. Z8Y & $0.184(4)$ & $29(1)$ & $0.371(3)$ & $61(1)$ & $2.0(2)$ & $0.99(6)$ & $32(2)$ & $6.4(3)$ & $0.50(3)$ \\
sint. Z3Y & $0.157(5)$ & $47(9)$ & $0.219(5)$ & $53(9)$ & & & & & $0.9(3)$ \\
sint. Z8Y & $0.180(1)$ & 100 & & & & & & & \\
mono. Z3Y & $0.168(1)$ & $85(2)$ & $0.26(1)$ & $15(3)$ & & & & & $6(1)$ \\
mono. Z9Y & $0.175(1)$ & 100 & & & & & & &
\end{tabular}

There were different points of view about the origins of the two shortest lifetime components in the nanopowder samples. In Ref. [3] the two components arise mainly from the annihilation of positrons inside nanograins, authors $[4,5]$ related these components to the GB's. In our paper [6], the latter interpretation was further supported by the observed $I_{2}: I_{1}$ ratios vs. the mean grain size. Positrons thus annihilate most likely in the vacancy-like defects at grain surface (the $\tau_{1}$ lifetime) or in a larger open-volume defects (probably triple points at GB intersections) with lifetime $\tau_{2}$.

The data on the sintered specimens shown in Table exhibit a pattern which is significantly different from that for the nanopowder materials and resemble more the results obtained for the corresponding monocrystals (see Table). This can be regarded as another argument favoring our above interpretation. The GB's contribution is indeed suppressed during sintering and positron annihilation in the grain interior, whose microstructure is obviously similar to that of the respective monocrystals, becomes of a dominating significance. On the other hand, the interpretation of lifetime $\tau_{1}$, observed for the monocrystalline specimens, as that of free positrons in the bulk is not straightforward. As shown in our recent 
study [10], slightly shorter bulk lifetimes are suggested by theoretical calculations for zirconia polymorphs. A more open-space structure of the lattice due to the non-stoichiometry-introduced oxygen vacancies in the YSZ system was ibidem tentatively considered as a possible cause for such a discrepancy. Indeed, the overall picture may become even more complex due to detrapping of positrons from the the oxygen vacancies and the other shallow defects.

Yet unclear point is also the nature of the $\tau_{2}$ components in the tetragonal monocrystal and sintered specimens. Assuming the two-state simple trapping model [11] the lifetime $\tau_{\mathrm{f}}$ calculated as $\tau_{\mathrm{f}}^{-1}=I_{1} / \tau_{1}+I_{2} / \tau_{2}$ from the data of Table should be equal to the bulk lifetime. The lifetime $\tau_{\mathrm{f}}=(177 \pm 1)$ ps obtained for the tetragonal monocrystal is close to the single component lifetime $\tau_{1}$ measured on the cubic one, see Table. This result indicates that, contrary to the cubic monocrystal, the tetragonal one contains open-volume defects which trap positrons and the lifetime $\tau_{1}$ is then a contribution of free positrons, while the longer lifetime $\tau_{2}$ comes from positrons trapped at the open-volume defects. For the sintered specimen Z3Y, similarly, $\tau_{\mathrm{f}}=(185 \pm 1)$ ps is comparable to the single-lifetime measured on the sintered cubic Z8Y specimen. These results indicate that the tetragonal YSZ specimens, investigated in the present work, contain additional open-volume trapping centers which are not occurring in the specimens with the cubic structure. Due to the above mentioned metastability of the tetragonal phase, however, the thermal history of the specimens is an important factor in this kind of studies. Misfit defects at phase boundaries can be considered as centers of positron trapping. Extended investigations seem to be necessary to clarify this picture.

An existence of pores is indicated by the $o$-Ps $\tau_{3}$ and $\tau_{4}$ components in the nanopowder specimens (see Table and refer to our recent paper [6] for more details). On the contrary to the nanopowder specimens, these $o$-Ps components were found to be absent in the sintered materials. The sintering process of ceramics, in which the surface energy is lowered, is accompanied by a volume shrinkage connected with the disappearance of pores. Obviously, our observations are consistent with such a picture.

A behavior of lifetime components resembling the differences between the nanopowder and sintered materials shown in Table was observed by Yagi et al. [5] who studied sintering process on the system of a slightly different composition, viz. the $\mathrm{ZrO}_{2}+2$ mol.\% $\mathrm{Y}_{2} \mathrm{O}_{3}$. In their work, sintering is seen by PAS as a gradual transformation of the two components in the $\tau<0.5$ ns region into the single-component lifetime spectrum $(\tau \approx 170 \mathrm{ps})$ and a vanishing of the $o$-Ps components above $1100^{\circ} \mathrm{C}$.

\section{Conclusions}

It is argued in the present work that positron annihilation in grain interiors dominates in the sintered YSZ's. This further supports our conclusions [6] that positrons annihilate mainly as trapped in defect structures associated with GB's 
in the compacted nanopowders. Present PL data also suggest that the process of sintering zirconia-based nanoceramics may be followed also via the shorter lifetime components $(<0.5 \mathrm{~ns})$ in PL measurements with sufficiently high resolution. Generally, the latter approach deserve a more attention in the future because it might bring a new information about the details of grain boundary evolution during the sintering ceramic materials.

\section{Acknowledgments}

This work was granted by the Ministry of Schools, Youths and Sports of the Czech Republic under scientific plan No. 0021620834, The Czech Scientific Foundation (contract No. GA106/06/0270) and the National Academy of Science of Ukraine (scientific plans No. A 116/06 H and A 106U006933).

\section{References}

[1] www.americanelements.com.

[2] K. Ito, Y. Yagi, S. Hirano, M. Miyayama, T. Kudo, A. Kishimoto, Y. Ujihira, J. Ceram. Soc. Japan 107, 123 (1999).

[3] Z. Wang, Z.W. Chen, J. Zhu, S.J. Wang, X. Guo, Radiat. Phys. Chem. 58, 697 (2000).

[4] J.E. Garay, S.C. Glade, P. Asoka-Kumar, U. Anselmi-Tamburini, J. Appl. Phys. 99, 024313 (2006).

[5] Y. Yagi, S. Hirano, Y. Ujihira, M. Miyayama, J. Mater. Sci. Lett. 18, 205 (1999).

[6] J. Cizek, O. Melikhova, J. Kuriplach, I. Prochazka, T.E. Konstantinova, I.A. Danilenko, Phys. Status Solidi C 4, 3847 (2007), DOI $10.1002 /$ pssc. 200675844 .

[7] T. Konstantinova, I. Danilenko, N. Pilipenko, A. Dobrikov, in: 9th Cimtec - World Ceramics Congress. Ceramics: Getting into the 2000's - Part A, Florence 1998, Ed. P. Vicenzini, Techna Srl., Faenza (Italy) 1999, p. 305; A.M. Slipenyuk, M.D. Glinchuk, I.P. Bykov, A.V. Ragulya, V.P. Klimenko, T.E. Konstantinova, I.A. Danilenko, Ferroelectrics 298, 289 (2004).

[8] F. Becvar, J. Cizek, L. Lestak, I. Novotny, I. Prochazka, F. Sebesta, Nucl. Instrum. Methods Phys. Res. A 443, 557 (2000).

[9] H. Surbeck, Helvetica Phys. Acta 50, 705 (1977).

[10] O. Melikhova, J. Kuriplach, J. Cizek, I. Prochazka, W. Anwand, G. Brauer, T.E. Konstantinova, I.A. Danilenko, Phys. Status Solidi C 4, 3831 (2007), DOI $10.1002 /$ pssc. 200675858 .

[11] P. Hautojärvi, C. Corbel, in: Positron Spectroscopy of Solids, Proc. Int. School of Physics "Enrico Fermi", Course CXXV, Varenna (Italy) 1993, Eds. A. Dupasquier, A.P. Mills, Jr., IOS Press, Amsterdam 1995, p. 491. 